\title{
Habitus y Práctica en la Escuela de Rehabilitación Humana de la Universidad del Valle desde la concepción de Bourdieu
}

Aida Josefina Rojas* María Helena Rubio**

\section{Resumen}

Este trabajo tiene el propósito de hacer una mirada retrospectiva y analizar desde la perspectiva de la teoría de Bourdieu las razones por las cuales se presentó la conformación de la Escuela de Rehabilitación Humana de la Universidad del Valle.

Se analiza desde la perspectiva de género nuestras profesiones y sus implicaciones. Se resalta la construcción y transformación de las prácticas en busca de la identidad y desarrollo de la Escuela de Rehabilitación Humana. Por otro lado se rescata la autonomía, equidad, transformación de las prácticas en habitus.

Palabras claves: Género, Roles, Habitus, Campo.

* Terapeuta Ocupacional U.V. Profesora Auxiliar Universitaria: Universidad del Valle.

** Terapeuta Ocupacional U.N. Especialista Docencia. Profesora Asistente Universidad del Valle. 


\section{Habitus and practice in the Human Rehabilitation School of the Universidad del Valle From the conception of Bourdieu}

\section{AIDA JOSEFINA ROJAS}

Occupational Therapy Universidad del Valle

Auxiliary teacher

Universidad del Valle

\author{
MARÍA HELENA RUBIO \\ Occupational Therapy \\ Universidad Nacional \\ Specialist in docent university \\ Universidad del Valle \\ Assistance teacher Universidad \\ del Valle
}

This paper has the purpose of making a retrospective look, and analyze from the Bourdieu perspective theory, the reasons by which we present the conformation of the Human Rehabilitation School of the Universidad del Valle.

We analyze from the perspective of gender, our professions and its implications. We rebound the building and transformation of the practice to find the identity and development of the Human Rehabilitation School. In the other hand, we want to ransom the autonomy, equity, and transform the practice in habitus.

\section{MARCO REFERENCIAL}

Según Lerer ${ }^{1}$ el género se refiere al carácter cualitativo e interdependiente de la posición de mujeres y hombres en la sociedad. Las relaciones entre ambos están constituidas en términos de las relaciones de poder y dominación que estructuran las oportunidades que ofrece la vida a mujeres y hombres... El concepto hace posible distinguir las diferencias sexuales, fundadas biológicamente entre mujeres y hombres, de las diferencias determinadas culturalmente entre las funciones recib:das o adoptados por mujeres y hombres respectivamente en una sociedad determinada. Las primeras son invariables, tienen carácter de destino. Las últimas se pueden transformar y pueden variar en función de influencias políticas y de la evolución de la opinión. Arias².

Los roles de género son el conjunto de expectativas, diferenciadas entre las de los hombres y las mujeres, sobre cómo ser, cómo sentir, cómo actuar, y sobre qué posibilidades se tienen dentro del grupo social. Estos roles son asignados por el 
grupo y son ejercidos por las personas según estos se asuman como hombres o como mujeres. Algunos de los criterios para diferenciar los roles en nuestra sociedad es el de género. Proequidad ${ }^{3}$.

Analizando desde la perspectiva de un desarrollo humano integral y equitativo, este proceso de asignación de roles que generalmente es aceptado como necesario para el crecimiento y el progreso de las sociedades, presenta la grave falla de ir acompañado de un proceso simultáneo de valoración diferencial entre unos roles y otros, que en consecuencia lleva a la subordinación de unos grupos a otros dentro de la sociedad.

En el análisis social, el concepto de roles de género es fundamental para comprender los procesos que se interrelacionan en la actuación social cotidiana:

1. El proceso mediante el cual el hecho de ser hombre o de ser mujer, se asocia naturalmente con algunas actividades, potencialidades, limitaciones y actitudes.

2. El proceso mediante el cual algunas actividades y expresiones se clasifican naturalmente como masculinas o femeninas .

3. El proceso mediante el cual los grupos sociales valoran de manera diferencial - las actividades identificadas como masculinas o femeninas.

4. El proceso mediante el cual la misma actividad es valorada de forma diferente dependiendo de si es realizada por un hombre o por una mujer. Proequidad ${ }^{3}$.

Las relaciones de género son dinámicas y susceptibles de transformarse a través de la interacción humana. Lo que un grupo social permite, promueve y espera de las mujeres, siempre estará relacionado con lo que permite, promueve y espera de los hombres, y viceversa. La categoría género, aborda esta dinámica de interdependencia y permite comprender si en un grupo social determinado estas relaciones son de complementariedad, subordinación o equidad. Calero ${ }^{45}$.

"Recién en el siglo IX las mujeres dejamos paulatinamente el analfabetismo que tanto favorecía a nuestros dominadores, lo cual nos permitió acceder a la cultura, en el siglo XX las mujeres encontramos nuevas y reales posibilidades de desarrollo, comenzamos a actuar con lucidez, a comprender el valor de acceder al autoconocimiento, a la educación y a la cultura, nos desarrollamos idóneamente accedemos a diversos puestos y posiciones". Lerer ${ }^{1}$. 
Desde la concepción de Bordieau ${ }^{6.7}$ Campo significa pensar en términos de relaciones. Puede definirse como una red o configuración de relaciones objetivas (independiente de la conciencia y la voluntad individual), entre posiciones, es un escenario de relaciones de fuerza y de luchas encaminadas a transformarlas y por consiguiente el sitio de un cambio permanente, posee su lógica, reglas, regularidàdes específicas. Todo campo constituye un espacio de juego cuyos límites son fronteras dinámicas. El Habitus lo define como un sistema socialmente constituido de disposiciones estructuradas y estructurantes. Los agentes sociales son el producto de la historia, esto, es de la historia de todo el campo social y de la experiencia acumulada en el curso de una trayectoria determinada en el subcampo considerado.

\section{LA TRANSFORMACIÓN DE LAS PRÁCTICAS GENERADORAS DE LA ESTRUCTURA DE ESCUELA REHABILITACIÓN HUNANA (E.R.H.)}

La intención es revisar la estructura hábitos y prácticas y demostrar en la práctica la posibilidad de una objetivación sociológica de la relación de la E.R.H. con su objeto de acción.

No es posible entender la dinámica de un campo (el movimiento humano, la comunicación humana, y la ocupación humana) sino mediante el análisis de su éstructura, y de la misma manera, tampoco podemos comprender esta estructura sin el análisis genético de su constitución y de las tensiones entre las posiciones que lo constituyen o entre este campo en su conjunto y otros campos en especial el de Medicina Física y Rehabilitación.

La lógica del ajuste de las disposiciones a la condición permite comprender que en este campo donde interactuaban especialistas en Medicina Física y Rehabilitación con terapeutas físicas, ocupacionales y del lenguaje, se generaran relaciones de dominantes-dominados. Como sucede habitualmente, se vivió una inversión ficticia de los valores dominantes produciendo la ficción del mundo social de la Rehabilitación, confirmando así a los dominados èn su subordinación y a los dominantes en su dominación con la apariencia de un apropiada dinámica de interacción.

Las relaciones de dominación de parte de quienes lideraban el Departamento de Medicina Física y Rehabilitación no daban la oportunidad a las terapeutas de apro- piarse de la condición como docentes, generar desarrollos en su campo, y tener un 
sentido de pertenencia hacia la institución a la cual estaban vinculadas lo que suscitó alguna forma de resistencia.

Los dominados en cualquier universo social, están siempre en condición de ejercer cierta fuerza: la pertenencia a un campo implica por definición, la capacidad de producir efectos en él, por lo mismo, el de provocar reacciones de exclusión por parte de quienes ocupan en él posiciones dominantes. Quienes dominan en un determinado campo están en posición de hacerlo funcionar en su beneficio, pero siempre deben tener en cuenta la resistencia, las protestas, la reivindicaciones y las pretensiones, "políticas" o no de los dominados. Por esta razón, a partir de la reflexión en los profesores de fisioterapia, fonoaudiología, terapia ocupacional, se genera una gran indisposición, y replantean sus roles como docentes, profesionales y gestores de la formación académica de los estudiantes, lo que propició la ruptura y en consecuencia cambios en los hábitus y prácticas del campo de la Rehabilitación.

El primer cambio, se dio en la jefatura lo que generó una reacción adversa a la transformación del hábitus, que trajo como consecuencia la pérdida de este logro y la involución a la antigua política de dirección. Los periodos de crisis, en los cuales los ajustes rutinarios de las estructuras subjetivas u objetivas son brutalmente trastornados, constituyen una clase de circunstancias donde la elección racional puede predominar por lo menos entre aquellos agentes que puedan, por así decirlo darse el lujo de ser racionales. Por que el campo subyace y orienta las estrategias mediante las cuales los ocupantes de dichas posiciones intentan, individual o colectivamente salvaguardar o mejorar su posición e imponer el principio de jerarquización más favorable a sus propios productos.

Por otro lado, con base en la teoría de hábitus podemos comprender esta situación, debido a que los estímulos y la estructura del campo puede generar prácticas diferentes e incluso opuestas; en virtud de las transformaciones de la situación dentro de la cual opera.

Coyuntural con la política gestada por la Reforma Curricular el campo pasa a ser un escenario de relaciones de fuerza y de luchas encaminadas a transformarlo, $y$ por consiguiente, a generar un cambio permanente. Motivados por esta situación surge la E.R.H. la cual, a través del conocimiento del campo donde esta inmerso, logra su identificación y define su singularidad, su originalidad, su punto de vista 
como posición, a partir de la cual se conforma su visión particular del mundo y del mismo campo.

A la luz de la teoría del habitus como principio unificador y generador de prácticas permite comprender que la durabilidad, la transferibilidad, y la exhaustividad de un habitus están estrechamente ligadas en la práctica. Es la razón, por la cual, las prácticas pedagógicas de los profesores dependieron inicialmente de la estructura dada por las posibilidades que aquellos le aseguraron, es decir, de su trayectoria social, académica y de las disposiciones ( habitus) que se constituyeron en la relación prolongada de dicha estructura.

Al constituirse como una nueva estructura la E.R.H. y en concordancia con los principios de la Reforma Curricular, los sistemas de enseñanza adoptados variaron de acuerdo a las características específicas de su campo y fue necesario producir y reproducir, por los propios medios, las condiciones académicas pertinentes al desarrollo de las diferentes profesiones que la constituyen (autorreproducción). El sistema de enseñanza tiende a informar y a formar a los futuros profesionales, a través del principio integral de Rehabilitación.

Es claro que se han formado nuevas prácticas, existen nuevos líderes que a su vez reproducen la dinámica de la dominación, que propiciaran nuevas tensiones y posiciones en el campo que generaran transformaciones en las prácticas y habitus con el correr de los años, ojalá para bien de nuestras profesiones, de nuestra institución y de la comunidad.

Recordemos que el habitus no es el destino que algunas veces se ha creído ver en él; sino, el producto de la historia. Es un sistema abierto de disposiciones enfrentado a un continuo de experiencias nuevas, $y$ en consecuencia, afectado sin cesar por ellas. La mayoría de las personas están estadísticamente destinadas a encontrar circunstancias similares, a las cuales originalmente moldearon sus habitus.

Finalmente es pertinente, al reconocer nuestras profesiones como aún de predilección femenina, propender por valorar equitativamente todos los roles, desde la perspectiva de género. Inculcar y demostrar el sentido de nuestras profesiones a través, de la construcción y transformación de las prácticas, de la generación de nuevos conocimientos, de la construcción de un eje disciplinar, del reconocimiento del campo del conocimiento en particular, del logro de la autonomía del ejercicio pro- 
fesional, el establecimiento de poder y jerarquía en el trabajo interdisciplinar y transdisciplinar, y la ampliación de los escenarios de las prácticas con influencia comunitaria y regional dentro del contexto nacional e internacional.

\section{BIBLIOGRAFÍA}

1. Lerer, M. L. La Dulce Espera de la Pareja. Editorial Sudamericana Planeta Bs. As. 1987. Pp. 28-30.

2. Arias, L.; Bonilla, N. Desarrollo Filogenético, Somático y Bases Neurofisiológicas y psicológicas de la Sexualidad. 1996. Universidad del Valle. Pp. $39-47$.

3. Proequidad. Concepto del Análisis de Género. Pp. 18-27.

4. Calero, A. Cultura, mujer y profesión: La Mujer en la Universidad Pública. Universidad del Valle Facultad de Educación. 1993. Pp. 5-24, 45-47..

5. Calero, A. La Otra Universidad. Ediciones Cecan, Cali, 1991. Pp. 57 - 94.

6. Bourdieu, Pierre. En Fundamentos Sociológicos para una Teoría de la Escuela de Américo Calero. Respuestas. "Por una Antropología Reflexiva" México: Grijalbo, 1995. Pp. 63-78.

7. Bourdieu, Pierre. En Fundamentos Sociológicos para una Teoría de la Escuela de Américo Calero. "Sociología y Cultura". Grijalbo, 1990. Pp. 79-99. 